\title{
Mössbauer Studies of Pathological Brain Tissues Affected by PSP Disease
}

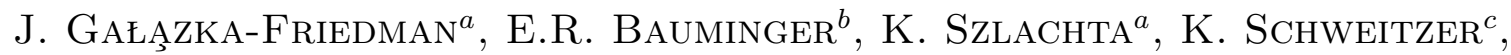 \\ Z. WszoleK ${ }^{c}$, D. DiCKson ${ }^{d}$ AND A. Friedman ${ }^{e}$ \\ ${ }^{a}$ Faculty of Physics, Warsaw University of Technology \\ Koszykowa 75, 00-662 Warsaw, Poland \\ ${ }^{b}$ Racah Institute of Physics, The Hebrew University, 91904 Jerusalem, Israel \\ ${ }^{c}$ Department of Neurology, Mayo Clinic, Jacksonville, FL, USA \\ ${ }^{d}$ Department of Neuroscience, Mayo Clinic, Jacksonville, FL, USA \\ ${ }^{e}$ Department of Neurology, Warsaw Medical University \\ Kondratowicza 8, 03-242 Warsaw, Poland
}

\begin{abstract}
Progressive supranuclear palsy (PSP) is a neurological disease leading to the damage of two brain structures: globus pallidus and substantia nigra. The pathomechanism of this disease is still unknown. One of the hypotheses is oxidative stress. Oxidative stress is an overproduction of free radicals in which iron may be involved. To verify the hypothesis that iron may play a role in PSP we performed the Mössbauer comparative studies of pathological and control tissues. Ten samples of PSP globus pallidus, ten samples of PSP substantia nigra, twelve control samples of globus pallidus and nine control samples of substantia nigra were measured in a conventional Mössbauer spectrometer at $90 \mathrm{~K}$. The Mössbauer spectra obtained for all samples showed well resolved doublets with an isomer shift of $0.46 \pm 0.01 \mathrm{~mm} / \mathrm{s}$ and a quadruple splitting of $0.70 \pm 0.02 \mathrm{~mm} / \mathrm{s}$. The main difference in these preliminary studies was in the concentration of iron. The concentration in PSP samples in globus pallidus was found to be $257 \pm 19 \mathrm{ng} / \mathrm{mg}$ tissue, compared to $183 \pm 22 \mathrm{ng} / \mathrm{mg}$ in control samples and $301 \pm 26 \mathrm{ng} / \mathrm{mg}$ in substantia nigra compared to $188 \pm 22 \mathrm{ng} / \mathrm{mg}$ in control samples. Taking into consideration that we did not notice any substantial increase in iron concentration in Parkinsonian substantia nigra compared to control substantia nigra, but a substantial increase in both substantia nigra and globus pallidus in PSP, may suggest that iron plays a different role in the pathomechanisms of PSP and of Parkinson's disease.
\end{abstract}

PACS numbers: 87.64.kx

\section{Introduction}

Progressive supranuclear palsy (PSP) is a neurodegenerative disorder with clinical symptoms of atypical parkinsonism. The pathological process affects various brain structures, mainly globus pallidus (GP) and substantia nigra $(\mathrm{SN})$. Both structures are shown in a schematic presentation of human brain (Fig. 1). In these brain structures accumulation of an abnormal protein (tau) is present. The pathomechanism of this neurodegeneration is not known and among other possible causes, iron mediated oxidative stress is considered. Iron in the brain under normal conditions is mostly located in a safe form within ferritin. Iron located outside of ferritin could trigger the Fenton reaction and initiate oxidative stress injury. Up to now there are only limited data regarding iron concentration in PSP tissues. We assessed the iron concentrations in two brain areas, which are involved in the pathological process - GP and SN - obtained from brains of patients who died with pathologically confirmed diagnosis of PSP. Both structures are known for many years to contain large concentrations of iron in normal human brains [1]. An increase in the total iron concentration in PSP SN was demonstrated in an early study using inductively coupled plasma spectroscopy [2], and the possibility that there may be an excess of iron in basal ganglia of patients suffering from PSP was suggested more recently by a study using electron nanodiffraction and high resolution electron microscopy [3].

For determining the iron concentration and iron binding compounds in globus pallidus and substantia nigra of patients who died with clinical diagnosis of progressive supranuclear palsy confirmed at autopsy we used the Mössbauer spectroscopy. This method was successfully used for iron determination in SN [4-7].

\section{Material and methods}

10 samples of GP and 10 samples of SN obtained from the same brains of PSP and 12 samples of GP and 9 sam- 


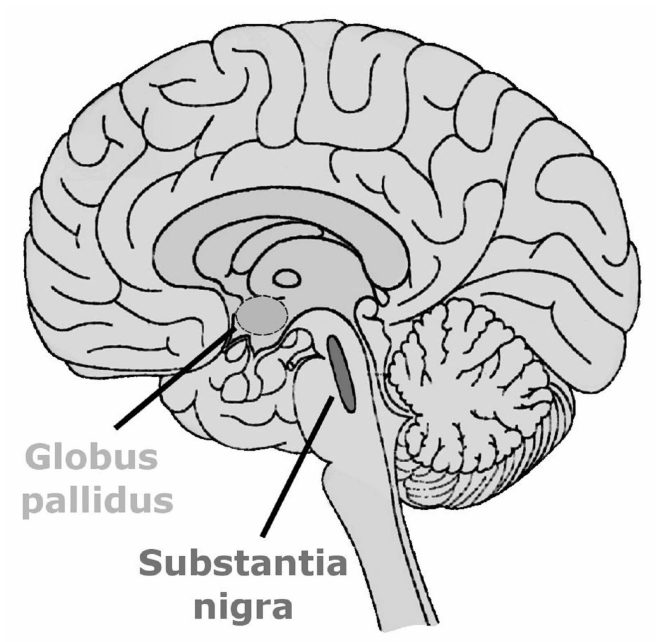

Fig. 1. Schematic presentation of human brain.

ples of SN from control age matched brains were evaluated. PSP was diagnosed in all cases based on pathological findings in the brain tissues. All PSP samples and 9 control SN samples were obtained from the Mayo Clinic (USA) and 12 GP control samples from the Warsaw Medical University. Fresh frozen samples dissected at autopsy were transferred to the Racah Institute of Physics in Jerusalem in dry ice. Here the samples were weighted and placed in plastic holders. All samples were measured at $90 \mathrm{~K}$ with a conventional constant acceleration Mössbauer spectrometer. A $100 \mathrm{mCi}{ }^{57} \mathrm{Co}$-in-rhodium source, giving a narrow unsplit emission line, was used. The velocity scale was calibrated using an $\alpha$-Fe absorber at room temperature. The $14.4 \mathrm{keV}$ gamma rays were detected using a proportional counter. The cryostat and counter were equipped with Al-coated Mylar windows. Each sample was measured for at least $48 \mathrm{~h}$. The experimental Mössbauer spectra were computer fitted with Lorentzian lines.

\section{Results and discussion}

The Mössbauer spectra obtained for all samples showed well resolved doublets with an isomer shift and quadrupole splitting equal within the errors for all measured samples (IS $=0.46 \pm 0.02 \mathrm{~mm} / \mathrm{s}$ and $\mathrm{QS}=$ $0.70 \pm 0.04 \mathrm{~mm} / \mathrm{s})$. These values are typical of ferritinlike iron. We could not detect in any of the measured samples any additional doublet, as described in a spectrum of GP by Dubiel et al. [8]. The Mössbauer spectra obtained from GP and SN from the same brain of a PSP patient are shown in Fig. 2. We determined the amount of iron in all samples from the overall spectral area. The calibration of the spectral area vs. the amount of iron was performed at $90 \mathrm{~K}$ with several frozen solutions of ferritin loaded with given amounts of iron.

After determining the amount of iron in the samples, the concentration of iron in the samples was calculated.

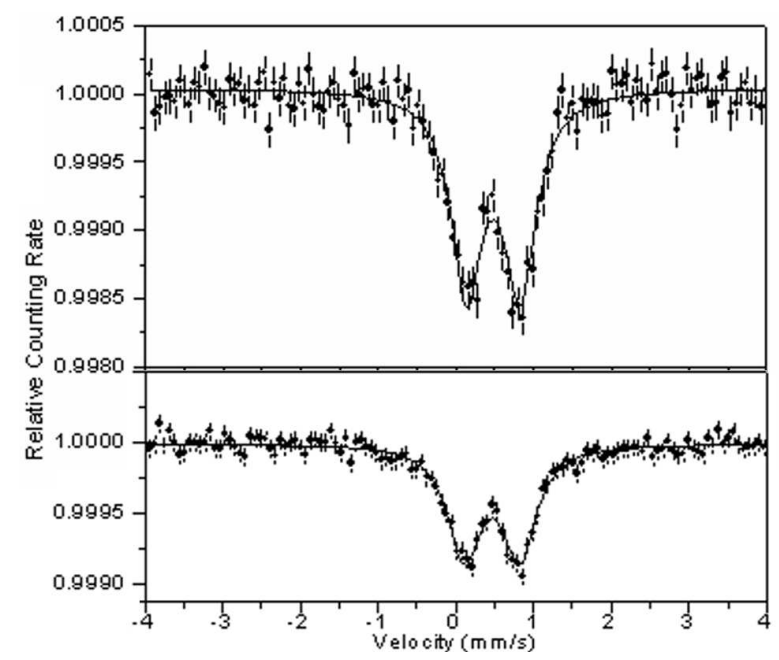

Fig. 2. Mössbauer spectra of PSP samples: bottom - SN sample (weight $=127 \mathrm{mg}$ ), top - GP sample (weight $=275 \mathrm{mg}$ ). The amount of iron in the samples is proportional to the spectral areas. Calculated from the respective spectral areas and the weights of the samples, the concentration of iron for this patient is $457 \mathrm{ng} / \mathrm{mg}$ GP tissues and $375 \mathrm{ng} / \mathrm{mg}$ SN tissues.

This calculation showed that the iron concentration in pathological tissues, both in SN and GP, was higher than in control tissues. The average iron concentration in PSP was $257 \pm 19 \mathrm{ng} / \mathrm{mg}$ in GP and $301 \pm 26 \mathrm{ng} / \mathrm{mg}$ in $\mathrm{SN}$, while in control brains the average iron concentration was $183 \pm 22 \mathrm{ng} / \mathrm{mg}$ in GP and $188 \pm 22 \mathrm{ng} / \mathrm{mg}$ in SN. The concentrations of iron in control GP and SN are similar to the values obtained by other methods [1]. The increase in the average iron concentrations in PSP samples compared to control is significant $(p<0.01)$.

Figure 3 shows the correlation between the concentration of iron found in globus pallidus and in substantia nigra of PSP patients.

The significant increase in the total iron concentration found by us in the preliminary measurements in both investigated structures, may suggest a role of iron in the degenerative process in PSP. It is interesting that in Parkinson's disease (PD), where also a role of iron in the neurodegeneration in $\mathrm{SN}$ is implicated, no difference within the limits of error in the total iron concentration between control and PD SN was found by the Mössbauer spectroscopy [6]. This difference between these two types of parkinsonism may reflect a different role of iron in these diseases. It may be postulated that in PD it is not the increase in total iron, but an increase in the non-ferritin iron, which causes the damage [7]. The significant increase in the total iron concentration in PSP both in SN and GP may be related to high iron loaded ferritin concentration within tau filaments [9]. An increase in the iron concentration in SN of PSP brains was also found by Dexter et al. [2]. These authors did not assess the iron level in GP of PSP patients. 


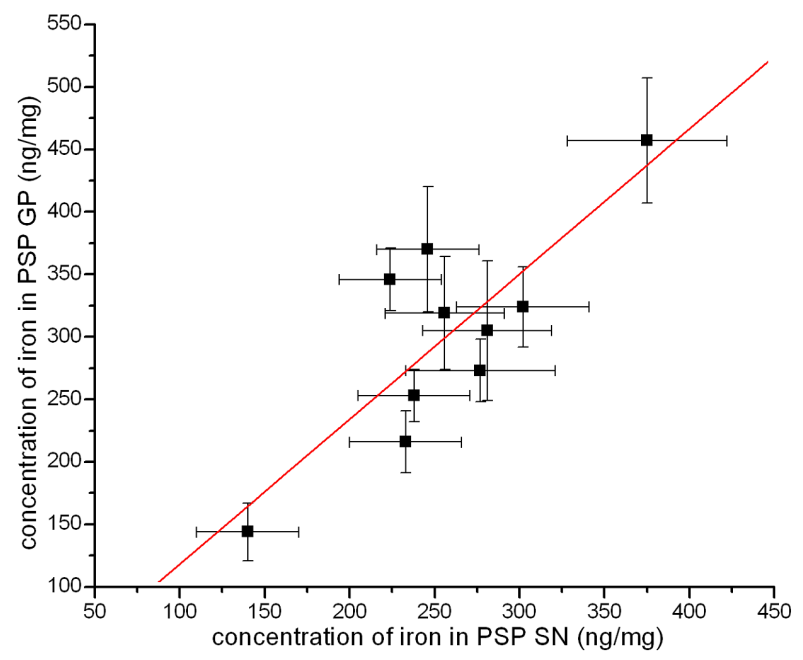

Fig. 3. Correlation of iron concentrations in globus pallidus and substantia nigra of PSP brains.

\section{Conclusion}

The preliminary results presented here, if confirmed by further studies, suggest that iron may be involved in the pathomechanism of nervous cells death not only, as believed, in Parkinson's disease, but also in PSP and possibly other neurodegenerative disorders.

The results of our study demonstrate that the Mössbauer spectroscopy is a useful method for investigating the possible role of iron in neurodegeneration. Taking into consideration that this method does not destroy the samples, the nervous tissues may be used later for other assessments.

\section{Acknowledgments}

This study was partially supported by a grant from the Polish Ministry of Science 2.R.07.P.W. (J.G.F., A.F., and K.S.).

\section{References}

[1] B. Hallgren, P. Sourander, J. Neurochem. 3, 41 (1958).

[2] D.T. Dexter, A. Carayon, F. Javoy-Agid, Y. Agid, F.R. Wells, S.E. Daniel, A. Lees, P. Jenner, C.D. Marsden, Brain 114, 1953 (1991).

[3] C. Quintana, J.M. Cowley, C. Marhic, J. Struct. Biol. 147, 166 (2004).

[4] E.R. Bauminger, M. Barcikowska, A. Friedman, J. Gałązka-Friedman, D. Hechel, I. Nowik, Hyperfine Interact. 91, 853 (1994).

[5] M. Gerlach, A.X. Trautwein, L. Zecca, M.B.H. Youdim, P. Riederer, J. Neurochem. 65, 923 (1995).

[6] J. Galazka-Friedman, E.R. Bauminger, A. Friedman, M. Barcikowska, D. Hechel, I. Nowik, Movement Disorders 11, 8 (1996).

[7] J. Galazka-Friedman, E.R. Bauminger, D. Koziorowski, A. Friedman, Biochim. Biophys. Acta 1688, 130 (2004).

[8] S.M. Dubiel, B. Zablotna-Rypien, J.B. Mackey, J.M. Williams, Eur. Biophys. J. 28, 263 (1999).

[9] M. Perez, J.M. Valpuesta, E.M. de Garcini, C. Quintana, M. Arrasate, J.L. Lopez-Carrascosa, A. Rabano, J.C. de Yebenes, J. Avila, Am. J. Pathol. 152, 1531 (1998). 